\title{
RECENSIÓN
}

\section{EDUCACIÓN PARA LA SALUD DEL SIGLO XXI. COMUNICACIÓN Y SALUD}

\author{
Coordinadora-editora. \\ $\mathrm{M}^{\mathrm{a}}$ Isabel Serrano González. \\ Díaz de Santos. Madrid. \\ Año 1997. \\ ISBN 84-7978-327-3. \\ 510 páginas.
}

Recensión: Isabel García García. Subdirección General de Epidemiología. Promoción y Educación para la Salud. Dirección General de Salud Pública. Ministerio de Sanidad y Consumo.

Cuatro secciones enmarcan este abultado y variado volumen sobre la EpS del siglo XXI. Variados son también los profesionales que participan (personal médico y de enfermería, psicólogos, educadores escolares y universitarios y trabajadores sociales), dejando patente la importancia de la multidisciplinariedad en el desarrollo de la Educación para la Salud (en adelante EpS).

La Sección primera, que engloba ocho capítulos, está dedicada a reflexionar sobre el marco teórico de la EpS, indispensable para entender la realidad y para poder situarnos ante los grandes cambios sociosanitarios acaecidos en nuestra sociedad actual y ante los que previsiblemente van a continuar dándose en el ya a las puertas siglo XXI.

Son tantas las materias tratadas en esta sección que prácticamente sólo podemos enumerarlas. Los movimientos migratorios, los grandes cambios producidos en las instituciones sanitarias, las nuevas enfermedades aparecidas en este siglo, los nuevos enfoques sobre salud publica, incluyendo la incorporación de los conceptos de promoción de salud, son temas que se analizan en este libro y sobre los que se reflexiona profundamente.

Las dimensiones culturales y los procesos de comunicación son aspectos tan importantes que es impensable poder trabajar en el campo de la promoción y EpS sin tenerlos en cuenta. Por ello, también se incluyen algunos capítulos sobre estos aspectos. Las relaciones con los demás y con el entorno es un proceso dinámico que hace del ser humano un ser en devenir, en permanente dialéctica con la realidad.

El bagaje ideológico, las creencias y modos de vida de las personas, su modo de comunicar y comunicarse consigo y con el entorno, el modo de actuar frente a la salud y la enfermedad son saberes que forman parte de la vida diaria, transmitidos en la relación del día a día, de manera no metódica, partiendo de la evidencia inmediata. El modelo cultural del que se parta tendrá claras incidencias sobre cómo educar a la población en salud. La EpS parte entonces tanto del saber cotidiano como del saber científico. Conocer y respetar el primero permite introducir conocimientos que llevan a la reflexión sobre el porqué de una acción, a razonamientos lógicos y al pasaje de actos intencionados positivos y saludables.

Y aquí podemos entroncar con las técnicas a utilizar para compaginar estos saberes. Se trata como bien se explícita en el capítulo 6 de esta primera sección, de las técnicas grupales principalmente. El proceso de educación para la salud pasa por la comunicación e interacción personal; una comunicación efectiva atraviesa el diálogo que se basa en hablar y escuchar con el fin de entender y hacerse entender. El trabajo de grupo permite la apro- 
piación de los saberes y experiencias de los otros y la posibilidad de comunicar y ofrecer los propios mediante la reflexión y discusión en grupo.

Dado que es el tema tratado en el último capítulo, aprovechamos la oportunidad de hablar acerca del logro que ha supuesto la inclusión de la EpS como materia transversal en el curriculum educativo. No vamos a caer en la tan manida afirmación de que el medio escolar es el más idóneo para desarrollar la EpS; también lo son el medio laboral y cualquier otro ámbito donde los distintos grupos de la comunidad viven y se desenvuelven habitualmente. Sin embargo, es cicrto que la escuela nos permite, desde el primer momento y de forma espiral, trabajar e incidir en la adquisición de actitudes y hábitos positivos para la salud así como comportamientos coherentes con el fomento de una vida saludable. Permite además poder incidir sobre toda la comunidad escolar incluidos padres y madres. Pero si bien es un paso importante que la EpS se recoja y reconozca en la LOGSE, no es menos cierta la necesidad de continuar apoyando, reforzando y estimulando el desarrollo de proyectos y acciones de educación para la Salud en el medio educativo. La existencia de una Red Europea de Escuelas Promotoras de Salud auspiciada por la Organización Mundial de la Salud y apoyada por organismos como la Comisión Europea o el Consejo de Europa y a la que España está adherida, pone de manifiesto la importancia de esta última afirmación.

En la sección segunda se tratan los problemas de salud que específicamente habrá que afrontar en el siglo XXI, en los que el papel de la EpS será imprescindible bien para atajarlos, bien para reducir el dolor y el sufrimiento que las mismas conllevan.

La salud mental también se incluye en este libro; este tema tan escasamente tratado habitualmente en promoción de la salud, es imprescindible si de verdad nos interesa el bienestar de las personas. Todavía seguimos aplicando nuestro esfuerzo en prevenir los aspectos físicos olvidándonos de algo tan esencial coino es el equilibrio emocional de las personas y el sentirse bien en el seno de una sociedad que se precie de ser saludable.

Las enfermedades crónicas y los accidentes de tráfico son también tratados en esta sección, teniendo presente los componentes individuales y sociales que actúan en ellos y por tanto la posibilidad de educar en estos aspectos.

La tercera sección se ocupa de un tema fundamental y sin embargo, muchas veces descuidado. Es la programación y planificación de las intervenciones en EpS. Las acciones educativas exigen de una adecuada programación que parta de un profundo conocimiento de la población a quien va a dirigirse la acción, de la delimitación y formulación del problema sobre el que se va a trabajar, de una estudiada definición de los objetivos que se pretende alcanzar así como de los contenidos y recursos disponibles y necesarios; la utilización de metodologías activas y participativas son imprescindibles sin olvidarnos, cosa que ocurre muy a menudo, de la necesidad de evaluar la pertinencia tanto del proceso como de los resultados de los programas aplicados.

En la sección cuarta, se expone el desarrollo concreto de proyectos llevados a cabo con grupos específicos como el de los niños y adolescentes diabéticos, enfermos terminales, grupos de autoayuda, grupos de mujeres, escuelas de padres y madres. Se incluyen en esta sección algunos capítulos sobre diversos recursos y técnicas que favorecen el desarrollo de la EpS.

Para finalizar, nos quedamos con una idea central desarrollada en este libro, es el papel que la EpS juega en la sociedad actual como respuesta a las necesidades de salud de los grupos y como el mejor medio de adquirir comportamientos positivos en relación con la salud individual y comunitaria y de modificar aquellos, contrarios al fomento de la salud. Este concepto de educación va unido al desarrollo de la capacidad de toma de decisiones conscientes y coherentes con un modo de vida saludable, de responsabilidad frente a la 
propia salud y la de los demás y de la adecuada utilización de los servicios sanitarios.

El desarrollo de la EpS es un indicador claro de calidad y bienestar de los pueblos. Desde esta realidad, se hace necesario no sólo profundizar en los conceptos, aunque sean prioritarios para avanzar, sino también una adecuada programación y planificación, eficaces metodologías, recursos, y sobre todo unos profesionales con una sólida formación en esta materia.
Quizás la conclusión a la que nos lleva este conjunto de trabajos sea que la EpS se puede aplicar en una realidad presente y palpable desde un enfoque integral basado en conocimientos sobre la persona y la realidad social actuales, desde una postura positiva hacia la sociedad y un reconocimiento de su capacidad para sortear los peligros y riesgos siempre que se les proporcionen las herramientas y recursos necesarios para ello. 